\title{
Synthesis and self-assembly of a carborane-containing $A B C$ triblock terpolymer: morphology control on a dual-stimuli responsive system
}

Roberto Fernandez-Alvarez, ${ }^{a}$ Eva Hlavatovičová, ${ }^{a}$ Krzysztof Rodzeń, ${ }^{\mathrm{b}}$ Adam Strachota, ${ }^{\mathrm{b}}$ Sami Kereïche, ${ }^{\mathrm{a}, \mathrm{c}}$ Pavel Matějíček, ${ }^{\mathrm{a}}$ Justo Cabrera -González, ${ }^{\mathrm{d}}$ Rosario Núñez ${ }^{\mathrm{d}}$ and Mariusz Uchman ${ }^{\mathrm{a}^{*}}$

${ }^{a}$ Department of Physical and Macromolecular Chemistry, Charles University, Hlavova 2030, 12843 Prague 2, Czech Republic

${ }^{b}$ Institute of Macromolecular Chemistry AS CR, v.v.i., Heyrovského nám. 2, 16206 Prague 6, Czech Republic

' Institute of Biology and Medical Genetics, First Faculty of Medicine, Charles University and General University Hospital in Prague, Purkynie Ustav, Albertov 4, 12801 Prague, Czech Republic

dInstitut de Ciencia de Materials de Barcelona (ICMAB-CSIC), Campus de la UAB, 08193 Bellaterra, Barcelona, Spain

*e-mail:uchman@natur.cuni.cz 


\section{Abstract}

Amphiphilic triblock terpolymers have attractive applications in the preparation of nanoparticles with controlled morphology. An additional level of morphology control can be provided by reactive blocks, whose interactions with the solvent vary under different stimuli. In this work, we synthesized a triblock terpolymer (poly(acrylic acid)-b-poly(4-hydroxystyrene)-b-poly\{1-[4-(1-methyl-1,2-dicarba-closododecaborane-2-yl methyl)-phenyl]ethylene\}) (PAA-b-PHS-b-PSC) containing carboranes as pendant groups by reversible addition fragmentation chain transfer (RAFT) polymerization and subsequently studied its behavior in aqueous solution. Once the nanoparticles were formed, the solubility of the second and third blocks was changed via $\mathrm{pH}$ and $\mathrm{CsF}$ reactions, respectively. The resulting micelles work as an ON/OFF system, using changes in fluorescence intensity at different pH values. 
Triblock terpolymer synthesis continuously broadens possibilities and product ranges in the field of solution-prepared nanoparticle architectures ${ }^{1}$ thanks to the high potential of terpolymers for new functional designs. ${ }^{2,3}$ Linear terpolymers are frequently designed to ensure different interactions between each block and the solvent and among the blocks (e.g.: hydrophilic, lipophilic, fluorophilic), thereby facilitating microphase separation of polymer chains in solution. Block order plays a key role in the formation of complex nanostructures and can lead to multi-compartment micelles. ${ }^{4,5} \mathrm{~A}$ powerful method for triblock terpolymer preparation is reversible addition fragmentation chain transfer (RAFT) polymerization. ${ }^{6,7}$ This living polymerization is compatible with various substrates and solvents, thus enhancing block length and polydispersity control. ${ }^{6,8,9}$ RAFT has also been used to prepare triblock terpolymers with different block orders and complex micellar structures. Therefore, RAFT is an excellent tool for introducing architectural flexibility into terpolymer nanostructures and for incorporating novel functional blocks.

Boron clusters are among the most resistant functional moieties incorporated into polymers to protect them from oxidation, ${ }^{10}$ heat ${ }^{11,12}$ and other degradation factors. Carboranes are commonly used in polymer chemistry, ${ }^{13}$ luminescent materials, ${ }^{14-19}$ and drug design ${ }^{20}$ thanks to their versatile and unique properties. Specifically in polymer chemistry, carboranes have been recently incorporated into vinyl-type polymers and transformed from closo- to nido- carboranes to modify the photoluminescent properties of these polymers. ${ }^{21,22}$ Despite these advances in carborane incorporation into linear polymers, their behavior and self-assembly in aqueous solution remains mostly unexplored limiting their possible application to thin film coatings. In contrast, non-cluster boron compounds are routinely incorporated into polymers and studied in solution. ${ }^{23-25}$ Hence, understanding their solution behavior is crucial for their use as stimuli-responsive blocks or in biomedical applications as agents in boron neutron cancer therapy treatment (BNCT).

The combination of incompatible blocks with different stimuli-responsive properties is a powerful tool for nanostructure manipulation. ${ }^{26}$ The availability of powerful polymerization techniques is widening the range of stimuli-responsive polymers based on creative designs. ${ }^{27,28}$ For example, closocarboranes can undergo deboronation into nido-carboranes in the presence of strong nucleophiles, which improves the water solubility. ${ }^{29}$ Thus, carboranes are excellent candidates for adding nucleophileresponsiveness to block copolymers.

Despite the progress in triblock terpolymer synthesis, in carborane incorporation into polymers and in stimuli-responsive nanostructure design, no stimuli-responsive triblock terpolymer with a caborane block has ever been prepared and studied in solution. Thus far, studies in solution have been limited to carborane dendrimers, ${ }^{30,31}$ and carborane copolymers have only been analyzed in solid state. Therefore, combining the structural flexibility of triblock terpolymers with the photoluminescent and protective properties of carboranes, through a simple synthetic approach such as RAFT, may enable us to study the self-assembly of these carborane nanostructures in solution.

Thus, in this study, we used RAFT polymerization to prepare poly(acrylic acid)- $b$-poly(4hydroxystyrene)-b-poly\{1-[4-(1-methyl-1,2-dicarba-closo-dodecaborane-2-yl methyl)-phenyl] ethylene)\}, (PAA-b-PHS-b-PSC) an amphiphilic triblock terpolymer. To our knowledge, this is the first study of a carborane-containing terpolymer in solution. This terpolymer was designed to have a charged block (PAA) which provides water solubility and micelle stability via electrostatic repulsion. The middle block (PHS) is ionizable but hydrophobic and requires a co-solvent for its solubilization in water. ${ }^{32,33}$ Lastly, a $^{2}$ 
short third block (PSC) has high hydrophobicity due to its bulky ortho-carborane moiety. The inclusion of carborane considerably differentiates PSC from PHS and PAA. Accordingly, low compatibility among blocks is expected. The volume fractions of the hydrophilic and hydrophobic blocks in PAA-PHS-PSC, estimated from the molecular weight of the corresponding blocks, 64\% (PAA) and 22\% (PHS) $+14 \%$ (PSC), respectively, were favorable to the assembly of the micelles with the hydrophilic shell and with the hydrophobic core, while the content of carborane monomer (5.7 wt.\% in elemental boron) suffice to lend the terpolymer valuable luminescent or "nuclear" (BNCT) properties. Furthermore, in our system, PHS and PSC can become more hydrophilic under different stimuli, namely $\mathrm{pH}$ and CsF, respectively. Thus, their interactions with the solvent can be individually controlled. For example, at $\mathrm{pH}>11$, the phenolic group of PHS is deprotonated, adding a delocalized charge, which increases hydrophilicity. When adding CSF and heating the solution, the PSC block undergoes deboronation, losing one $\mathrm{B}-\mathrm{H}$ vertex, and the resulting nido-cluster has an overall -1 charge. The preparation of such terpolymer is outlined in Figure 1a.
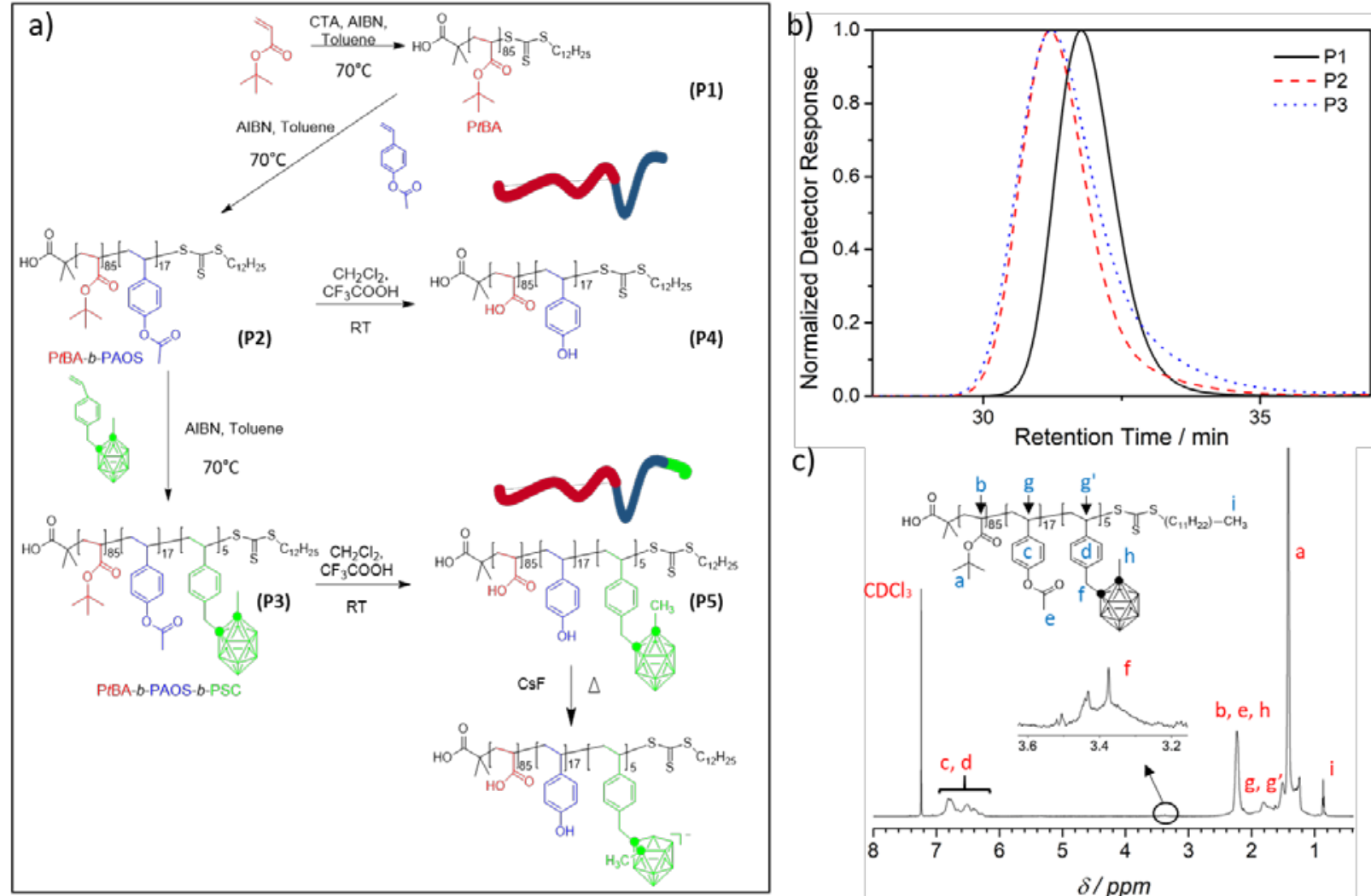

c)

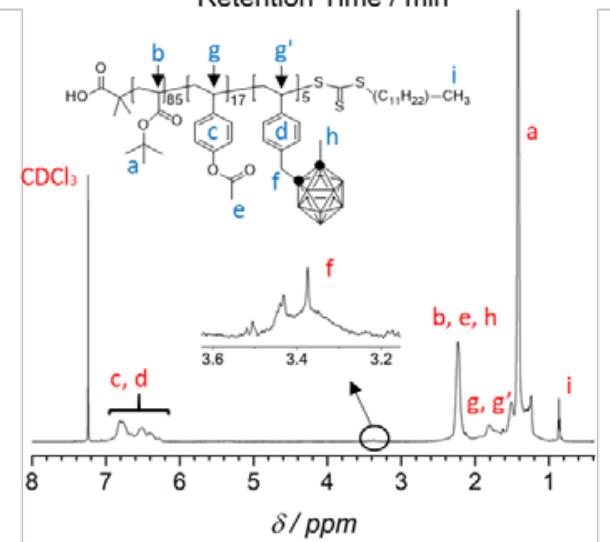

Figure 1. (a) Synthesis route to di- and tri-block amphiphilic copolymers, via RAFT polymerization followed by acidolysis. (b) SEC traces of PtBA (P1), PtBA-b-PAOS (P2) and of PtBA-b-PAOS- $b$-PSC (P3). (c) ${ }^{1} \mathrm{H}$ NMR spectrum of PtBA- $b$-PAOS- $b$-PSC (P3) in $\mathrm{CDCl}_{3}$ with signal assignment.

Poly(tert-butyl acrylate) P1 was synthesized as the first block of the future copolymers using 2,2'-azo-bisisobutyronitrile (AIBN) as the initiator and 2-(Dodecylthiocarbonothioylthio)-2-methylpropionic acid as the chain transfer agent (CTA) (Figure 1a). The RAFT agent/initiator combination was carefully selected to ensure reasonable polymerization rates for both acrylic and styrenic monomers. ${ }^{34}$ The polymerization 
was slower in case of the acetoxystyrene than in the case of tert-butyl acrylate and even slower for the sterically hindered and rigid styrene-based SC (further details are shown in Fig. S7) Toluene was used as the solvent to avoid an excessive increase in viscosity during polymerization, which resulted in relatively high monomer conversions without solidification. After removing the monomer residues, P1 (PtBA) was used as the macrotransfer agent for chain extension with 4-acetoxystyrene, yielding the diblock copolymer P2. After isolating P2, the carborane-containing monomer (SC) was added to the chain, thereby generating the triblock terpolymer P3. To more easily control the block length, and to add the most expensive block in the last step, the terpolymer was grown with the following order of blocks: PAAPHS-PSC. Lastly, acidolysis of the pendant ester groups of P3 yielded the amphiphilic P5, which was purified by dialysis. Similarly, the carborane-free diblock copolymer $\mathbf{P 2}$ was converted into the copolymer $\mathbf{P} \mathbf{4}$ by acidolysis, for comparative studies.

The final products of each synthesis step were analyzed by ${ }^{1} \mathrm{H}$ NMR and by SEC to assess their structure and molecular mass. Figure $\mathbf{1 b}$ and Table S1 show SEC results with monomodal distribution and relatively narrow molecular mass distributions in all polymers. Nevertheless, their dispersity increased with the successive addition of blocks. Additionally, a small tail was observed in the region of low molecular mass, which commonly occurs in RAFT copolymerization products. ${ }^{35,36}$ Both the apparent decrease in the $M_{n}$ of P5 and the discrepancies between $M_{n}(S E C)$ and $M_{n}\left({ }^{1} H\right.$ NMR) can be explained by differences in chain conformation between di- or triblock and polystyrene standards used for SEC calibration.

In turn, $M_{n}(N M R)$ were obtained from the ${ }^{1} \mathrm{H}$ NMR spectra (Figure 1c, details for $\mathbf{P 1}$ and $\mathbf{P 2}$ in Figure S1), using the ratios of suitable signal integrals from repeating units. The signal from the $\omega$-methyl group of the CTA at 0.86 ppm was used as "end-group-signal". The following signals were used: for PAA, the methine protons on the main chain at $2.2 \mathrm{ppm}$; for PHS, the signals of the aromatic protons near 6.6 ppm; lastly, the length of PSC was calculated using the wide signal around $3.3 \mathrm{ppm}$, which corresponds to the methylene bridge between styrene and the carborane cage in SC. The aromatic signals of the PHS block fully overlap with the aromatic signals of the PSC block. To evaluate the PHS block length of P3, the expected aromatic signal intensity (integral) from PSC was calculated as the double value of the signal integral at $3.3 \mathrm{ppm}$. This value was then subtracted from the integral of the signals near $6.6 \mathrm{ppm}$, thus yielding the remaining aromatic signal intensity from the PHS. After deprotection of the PAA and PHS blocks with trifluoroacetic acid (TFA), the polymers were analyzed via ${ }^{1} \mathrm{H}-\mathrm{NMR}$ to ensure the integrity of the samples. Upon deprotection, the broad signal corresponding to the $t$-butoxy group (1.4 ppm) disappeared, which improved the allocation of signals in that area (Figure S3). Signals at 12.5 and 9 ppm indicate the presence of carboxylic and phenolic protons, respectively, thus showing a successful deprotection. ${ }^{19} \mathrm{~F}-\mathrm{NMR}$ was acquired to rule out the presence of TFA, and only traces of TFA were detected in the $\mathbf{P 4}$ and $\mathbf{P 5}$ samples (further details and Figure $\mathbf{S 4}$ in $\mathrm{SI}$ ). Solid-state ${ }^{13} \mathrm{C}$ - and ${ }^{11} \mathrm{~B}-\mathrm{MAS}-\mathrm{NMR}$ spectra of P4 and P5 were also acquired to confirm the structure of the polymers and the integrity of the carborane cage (Figure $\mathbf{S 5}$ and $\mathbf{S 6}$, respectively).

After synthesis, we assessed differences in self-assembly behavior between P4 and P5 in various THF-water mixtures. The chain composition of P4 and P5 has a large portion of PAA (0.72 and 0.62 mass fraction, respectively), which indicates that spherical micelles should be the predominant morphology in aqueous solution. However, the resulting morphology can be controlled using selective solvents with different compositions. ${ }^{37,38}$ Accordingly, water was chosen as a solvent for the PAA block and as a non- 
solvent for the PHS and PSC blocks, whereas THF was used as a good solvent for PHS and PSC. As observed in similar systems, the nanoparticles are in a frozen state with entrapped morphology in waterrich mixtures. P4 and P5 were solubilized in solvent mixtures ranging from 10-90\% v/v THF-water. Unexpectedly, P4 was only soluble in a 90\% THF mixture, despite having a shorter hydrophobic part (no PSC block), whereas P5 was soluble in the entire range of mixtures. The ability of PHS to form intermolecular hydrogen bonds not only with itself but also with the PAA block may contribute to the insolubility of P4 in lower THF mixtures, and the presence of PSC may disrupt the hydrogen bonding in the case of P5, facilitating microphase separation even at high water content mixtures. The results from dynamic light scattering (DLS) indicate that both $\mathbf{P 4}$ and $\mathbf{P 5}$ form micelles in $\mathrm{THF}-\mathrm{H}_{2} \mathrm{O}$ mixtures and that their size and dispersity increase with THF v/v \% (Figure S4). Therefore, the resulting micelles consisted of one or two blocks (PHS and PSC) nanoconfined in a core surrounded by a charged PAA corona, as determined by zeta potential measurements (Table S7).

To understand the behavior of the linear polymers in water, the initial micellar solutions (THF$\mathrm{H}_{2} \mathrm{O}$ mixtures) were added dropwise to a large excess of water to entrap the micellar structures formed in the organic solvent mixture. ${ }^{39-41}$ Subsequently, samples were dialyzed against water. One (90\% THF, henceforth P4-90) and three (10, 50 and 90\% v/v THF-water; P5-10, P5-50, P5-90) solvent mixtures were chosen to further study P4 and P5 self-assembly in water, respectively. When micelles in a solvent mixture are added to a large excess of water, THF quickly migrates from the core towards the bulk solvent. ${ }^{42}$ Moreover, micelles become kinetically trapped, despite the preferential sorption of THF traces in micelles, if their core has a high value of glass transition $\left(T_{\mathrm{g}}\right)$, which PHS has. ${ }^{39}$ To further analyze the resulting micelles and their structure, we used DLS, cryo-TEM and SAXS.
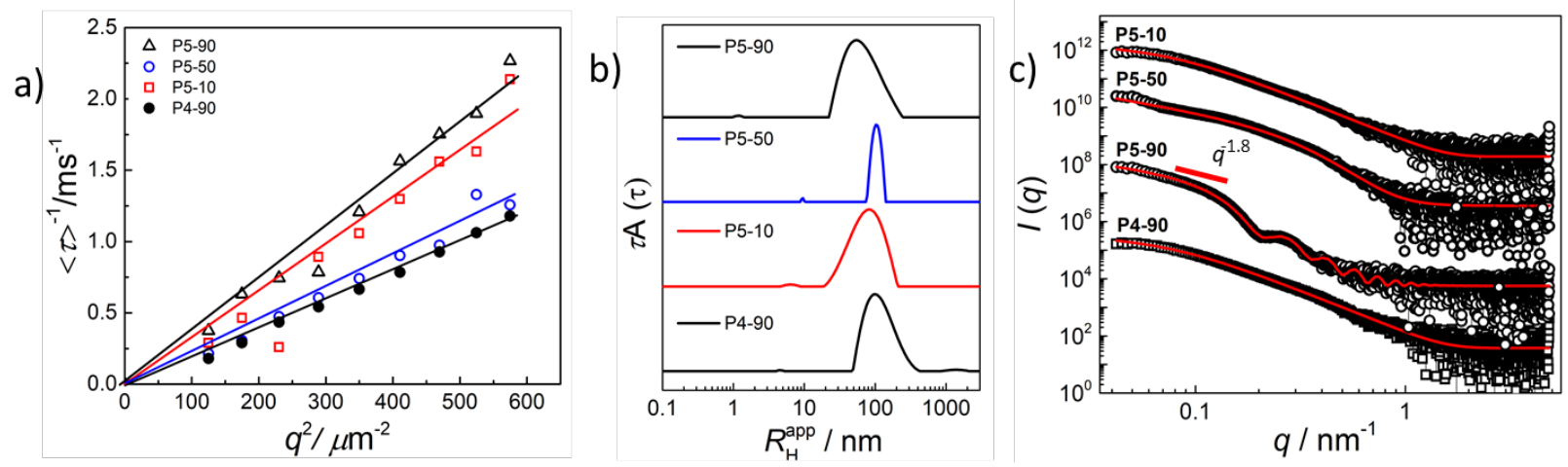

Figure 2. (a) Mean relaxation rates as a function of $q^{2}$, (b) DLS CONTIN distributions of apparent hydrodynamic radii (scattering angle, $\vartheta=90^{\circ}$ ), and (c) SAXS curves for the different micellar preparations of PAA- $b$-PHS (P4) and PAA- $b$-PHS- $b$-PSC (P5) in water. SAXS curves were independently fitted to their specific models (red line, details in SI). All SAXS curves, except P4, are artificially offset on the $\mathrm{Y}$ axis for clarity.

DLS measurements show monomodal distributions in all samples, with no large aggregates (Figure 2b, Table S6). P4-90 produces larger micelles than all P5 samples, which indicates the formation of a less compact and partly swelled PHS micellar core. Micelle stability in water is determined by the charged PAA corona, as shown by $\zeta$-potential measurements $(\zeta>-35 \mathrm{mV}$, Table S7). Although some deviations were observed in P5-90, which could be attributed to the inner motion of long cylindrical 
micelles (Figure 2a), the linear correlation between the mean relaxation rates $(\tau)$ and $q^{2}$ confirms that $\tau$ corresponds to the diffusive motion of micelles.

Cryo-TEM confirmed the presence of spherical micelles, as expected for both P4 and P5 (Figure 3). However, P5 showed variations in shape caused by the original selective solvent composition. Both P5-10 and P5-50 form spherical micelles, whereas P5-90 formed cylindrical micelles due to increased core swelling (Figure 3 and Figure S14). This volumetric increase in the core reduces the curvature of the micelles at the interface and allows them to acquire a cylindrical shape. Comparatively, in the same solvent system, P4-90 forms only spherical micelles, thus indicating that the bulky carborane block of P5 significantly contributes to the volume fraction of hydrophobic parts. Figure $\mathbf{3}$ also depicts the possible shapes of the micelles obtained and the distribution of the particular blocks in the nanoparticles. Thus, SAXS was used to further study the structure of the micelles (Figure 2c). The models used for fitting SAXS curves are listed in SI.

The SAXS curves of P4-90 micelles were fitted with a Pedersen-Gertenberg form factor consisting of hard spherical core and a corona of gaussian chains attached to the core. These estimated values of core radius were very similar to those assessed by cryo-TEM $\left(R_{\text {core }} \approx 7.8 \mathrm{~nm}\right)$, which indicates an adequate choice of fitting model (Table S5). In conclusion, P4-90 micelles are predominantly spherical.

The SAXS curves of P5-90 were fitted using a combination of long cylinder and sphere form factors. These SAXS curves clearly indicate cylindrical micelles because the power law exponent has a value of -1.8 at low $q$ values. Although the power law exponent of cylindrical micelles is typically $\approx-1$, at low $q$-range, the slight deviation may be explained by the concomitant presence of spherical and cylindrical micelles in P5-90. The fitting model provides cylinders with a cross section radius of $20 \mathrm{~nm}$ and spheres with a radius of $17 \mathrm{~nm}$, which is in excellent agreement with the cryo-TEM results (Table S3, Figure $\mathbf{2} \mathbf{b}$ and $\mathbf{3}$ ).

Conversely, our initial attempts to fit P5-10 and P5-50 to the form factor used for P4-90 or to simple spherical models failed. We expected PSC to form distinct compartments due to incompatibility with the PHS core. To explore this possibility, we fitted the P5-10 SAXS curve to a model consisting of small scattering objects (representing compartments or carborane clusters) with attractive forces (simulated by a mass fractal structure factor). The model yields $0.4 \mathrm{~nm}$ spherical domains and fractal aggregate of $13 \mathrm{~nm}$ size (Table S4). Therefore, the short PSC block may form compartments inside the PHS core. However, the fit was not entirely satisfactory at high $q$ values. In contrast, the Fisher-Burford cluster model provided good fits. The apparent "shapeless" nature of P5-10 and P5-50 SAXS curves derives from significant dispersity of polymers and corresponding micelles (Table S1). Nonetheless, the values from the Fisher-Burford model agree well with the results from cryo-TEM and DLS. The $R_{\mathrm{g}}$ values obtained from the SAXS fit were in the range of $R_{\text {core }}<R_{\mathrm{g}}<R_{\mathrm{H}}$ (Table S2). In addition, the power law exponent values of both P5-10 and P5-50 indicate that these micelles have a rough surface. 


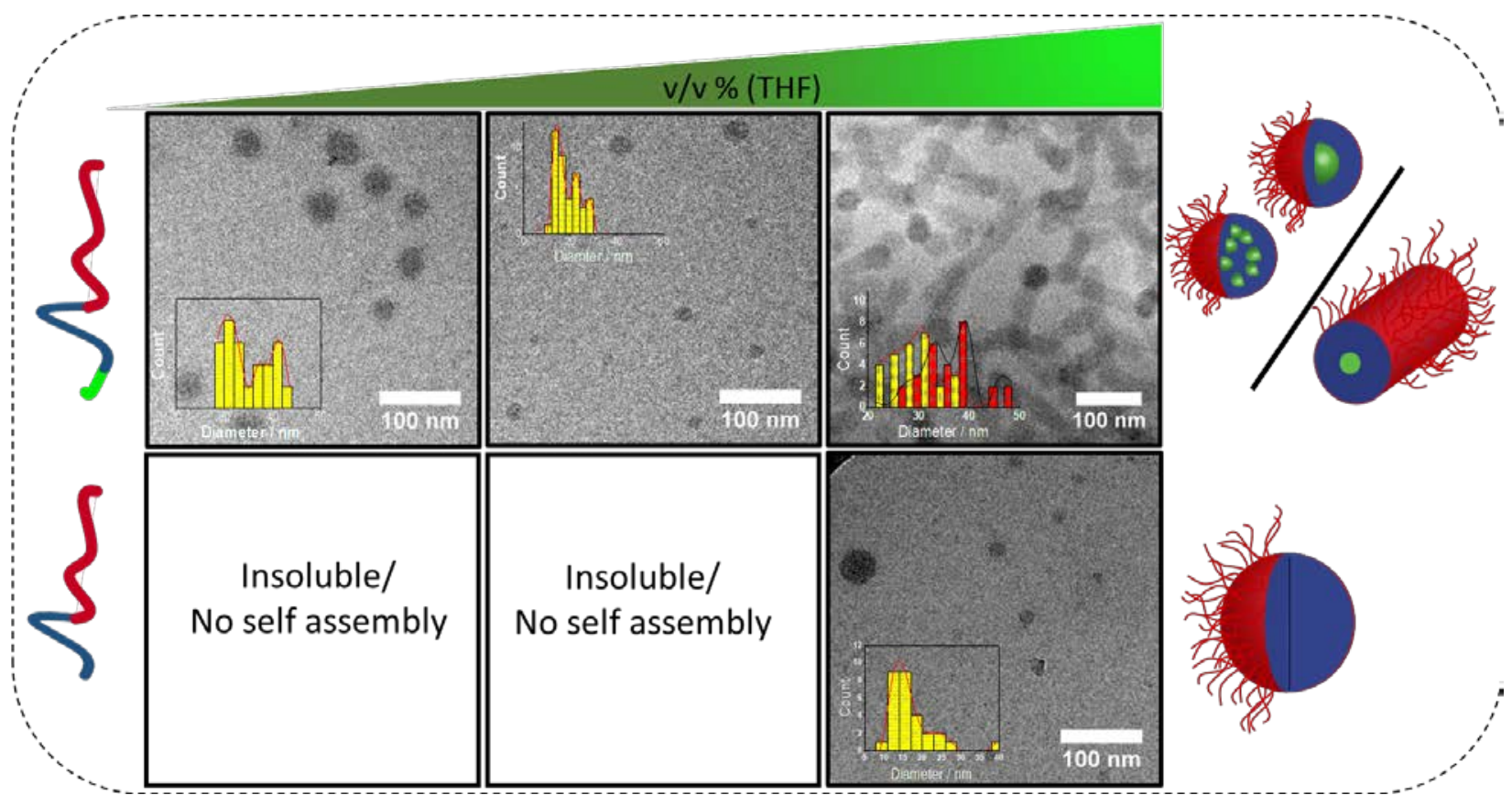

Figure 3. Cryo-TEM pictures depicting self-assembly structures of PAA- $b$-PHS (P4) (bottom) and PAA- $b$ PHS- $b$-PSC (P5) (top) in water. The volume percentage of THF refers to the percentage used to prepare the micelles (THF-water $\mathrm{v} / \mathrm{v} \%$ ). Inset: Histogram depicting the size distribution of spherical micelles (yellow) and the cross-section diameter of cylindrical micelles (red).

Lastly, we modified the solvophobic interactions between the PHS and PSC blocks in P5-10 micelles by changing the $\mathrm{pH}$ or alternatively by treating them with CsF, thus increasing their hydrophilicity in both cases, to assess the resulting structural changes. These changes were analyzed by TEM, DLS and fluorescence spectroscopy. The results showed that the increase in $\mathrm{pH}$ affects the core size of the micelles, whereas the fluoride-treatment changes the inner structure of the core (Figure 4).

When increasing the $\mathrm{pH}$ to $12\left(\mathrm{p} K_{\mathrm{a}}(\mathrm{PHS})=9-11.5\right)^{43}$ to ensure a high degree of deprotonation, the PHS block became charged (unlike the PSC block), increasing the hydrophilic character. DLS measurements showed that $R_{\mathrm{H}}^{\text {app }}$ increased from $74 \mathrm{~nm}$ to $114 \mathrm{~nm}$ because the corona extension. In addition, TEM images were acquired to track changes in the micelle core. The samples were treated with a variety of staining methods $\left(\mathrm{UO}_{2}\left(\mathrm{CH}_{3} \mathrm{COO}\right)_{2}, \mathrm{RuO}_{4}\right.$ and $\mathrm{OsO}_{4}$; details in $\left.\mathrm{SI}\right)$ to improve the compartments visualization. All staining methods proved effective in distinguishing the core and the corona (Figure 4a, Figures S16 and S17). The TEM images show a clear reduction in core size after the increase in $\mathrm{pH}$, thus indicating that PHS is deprotonated and becomes part of the corona (Figure 4a, histogram).

In the fluorescence measurements, P5-10 samples showed two distinctive fluorescence bands: One around $308 \mathrm{~nm}$ attributed to emission from single repetitive units of PHS and a broad band around $350 \mathrm{~nm}$ resulting from excimer emission of $\mathrm{PHS} .{ }^{44}$ However, the increase in $\mathrm{pH}$ to $\mathrm{pH}>\mathrm{p} K_{\mathrm{a}}(\mathrm{PHS})$ causes an abrupt loss of fluorescence, which proves that PHS block is the main contributor to the fluorescence signal. This fluorescence was restored by lowering the $\mathrm{pH}$ to $\mathrm{pH}<\mathrm{p} K_{\mathrm{a}}$ (PHS) (Figure 4c). Moreover, this ON/OFF process can be repeated without affecting the spherical shape of the micelles thanks to the 
presence of the hydrophobic PSC block. Therefore, we determined that changes in $\mathrm{pH}$ can work as an OFF/ON switch for fluorescence in P5 micelles.

When adding CsF for the deboronation of the closo-carborane clusters, the PSC block became charged. Furthermore, this reaction allowed us to selectively change the solvophobic character of this block without affecting the PHS block. After $24 \mathrm{~h}$, DLS measurements showed a small decrease in $R_{\mathrm{H}}{ }^{\text {app }}$ (8 $\mathrm{nm})$. Deboronation was confirmed via ${ }^{11} \mathrm{~B}\left\{{ }^{1} \mathrm{H}\right\}$ NMR spectroscopy, and the quadruplet signal at $0.6 \mathrm{ppm}$ is a known side product of deboronation when using CSF. Post-deboronation TEM images revealed that the micellar shape and the core size remained almost unchanged (Figure 4a), thus indicating that the PSC block remains in the core, despite the increase in its hydrophilicity. In addition, we observed a $27 \%$ increase in fluorescence intensity. We speculate that carborane moieties are distributed in the PHS core and that they disrupt the hydrogen-bonding network of the PHS block before deboronation. After deboronation, PHS and PSC blocks undergo a micro-phase separation in the core, which allows the formation of a more extensive hydrogen bond network among PHS chains, thus increasing the rigidity of the PHS compartments and the fluorescence intensity. ${ }^{44}$ Accordingly, we added a small amount of THF to the reaction mixture to increase core plasticity and to promote the rearrangement of PSC domains. In this case, fluorescence intensity increased by $84 \%$ (after removal of THF) (Figure 4c and Figure S2). A control sample containing both P4 and P5 micelles was prepared to ensure that the observed fluorescence effect resulted from deboronation and not from other effects (Figure S3). Therefore, P5 micelles can act as a responsive system capable of lowering (high $\mathrm{pH}$ ), restoring (neutral $\mathrm{pH}$ ) or increasing ( $\mathrm{CsF}$ ) the fluorescence intensity in response to external stimuli, while maintaining their stability and integrity.
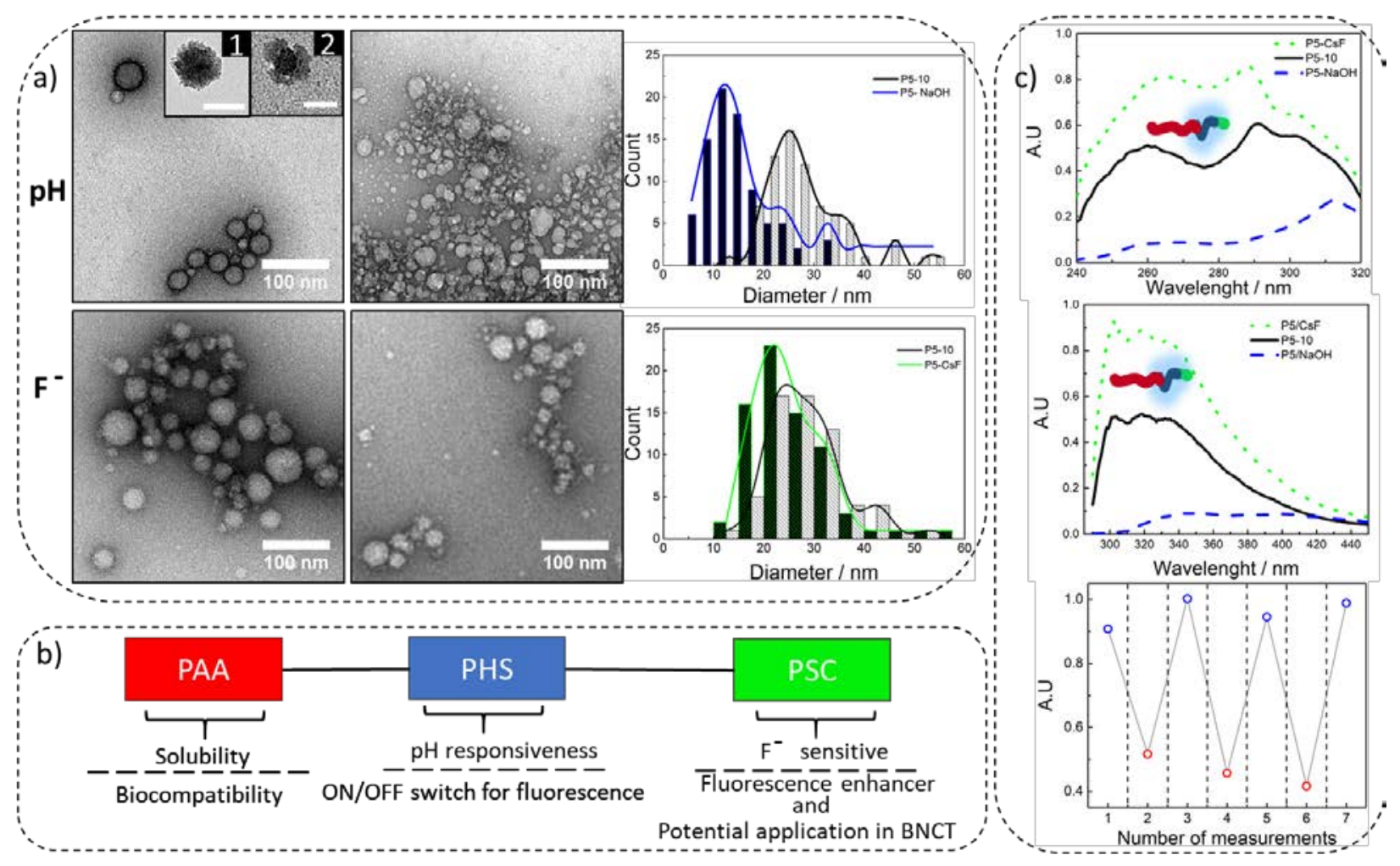
Figure 4. (a) Upper panel: TEM micrograph of P5-10 at neutral pH (left) and at basic pH (center) after $\mathrm{UO}_{2}\left(\mathrm{CH}_{3} \mathrm{COO}\right)_{2}$ staining, (right) histogram depicting the number size distribution of the core diameter. Inset: Detailed micelles stained with $\mathrm{RuO}_{4}(\mathbf{1})$ and $\mathrm{OsO}_{4}(2)$ in comparison with the micelle after uranylstaining; the scale bar corresponds to $50 \mathrm{~nm}$. Bottom panel: TEM micrograph before (left) and after (center) reaction with $\mathrm{CsF}$, (right) histogram depicting the number size distribution of the core diameter. (b) Schematic representation of triblock terpolymer and function of individual blocks. (c) Excitation spectra $\left(\lambda_{\mathrm{em}}=350 \mathrm{~nm}\right.$ ) (top), fluorescence spectra $\left(\lambda_{\mathrm{ex}}=274 \mathrm{~nm}\right.$ ) (middle) of P5-10 micelles demonstrating fluorescence response to different stimuli. Fluorescence intensity of P5-10 micelles at pH 11.8 (red) and 8 (blue) working as an ON/OFF switch (bottom).

In conclusion, we used RAFT polymerization to synthesize a novel triblock terpolymer containing carboranes: (poly(acrylic acid) -b- poly(4-hydroxystyrene) -b- poly\{1-[4-(1-methyl-1,2-dicarba-closododecaborane-2-yl methyl)-phenyl] ethylene\}), $\left(\mathrm{PAA}_{85}-b-\mathrm{PHS}_{17}-b-\mathrm{PSC}_{5}=\mathrm{P} 5\right)$. The amphiphilic terpolymer self-assembles in water/THF mixtures, and the micelles maintain stability when subsequently transferred into water. The morphology of the resulting micelles was controlled by core swelling with THF during the first preparation step achieving a transition from spherical to cylindrical micelles. Moreover, the inclusion of PHS and PSC blocks into the terpolymer granted control over the photophysical properties of the micelles and their use as a dual responsive system to both $\mathrm{pH}$ and fluoride anions. Specifically, we were able to use $\mathrm{pH}$ stimulus as an OFF/ON fluorescence switch in P5 micelles, and fluoride sensitivity amplified fluorescence through modification of the micellar core structure. Therefore, we developed a new method to incorporate carboranes into a terpolymer system capable of self-assembling into stable nanoparticles with a biocompatible stabilizing corona suitable for future applications in the design of boron nanocarriers for boron neutron capture therapy.

\section{Author Contributions}

$\mathrm{MU}, \mathrm{RF}-\mathrm{A}$, and KR designed research. RF-A and $\mathrm{EH}$ performed physicochemical characterization of polymer. KR synthesized the polymer. RN and JC-G synthesized carborane monomer. RF-A, EH, AS, KR and PM analyzed data. RF-A, PM and MU wrote the manuscript.

\section{Acknowledgments}

The authors acknowledge the financial support from the Czech Science Foundation Grant No. 17-00289Y. Authors would like to thank Prof. Miroslav Štěpánek for his counseling in SAXS fitting and interpretation, Prof. Jiři Vohlídal for his assistance in polymer nomenclature, Dr. Jiří Brus (IMC, Prague) for solid state NMR measurements, and Dr. Carlos V. Melo for editing the manuscript. The group from ICMAb thanks to Spanish Ministerio de Economía y Competitividad, MINEICO (CTQ2016-75150-R9 and and Generalitat de Catalunya (2017/SGR/1720) for the financial support.

\section{References}

(1) A.Blanazs, S. P. Armes and A. J. Ryan, Macromol. Rapid Commun., 2009, 30, 267-277.

(2) S. Ludwigs, A. Böker, A. Voronov, N. Rehse, R.Magerle and G. Krausch, Nat. Mater., 2003, 2, 744747.

(3) X. Cao, W. Mao, Y. Mai, L. Han and S. Che, Macromolecules, 2018, 51, 4381-4396. 
(4) L. Verheyen, B. Timmermans and G. Koeckelberghs, Macromolecules, 2018, 51, 6421-6429.

(5) A. O. Moughton, M. A. Hillmyer and T. P. Lodge, Macromolecules, 2012, 45, 2-19.

(6) G. Moad, E. Rizzardo and S. H. Thang, Polymer, 2008, 49, 1079-1131.

(7) S. Perrier, Macromolecules, 2017, 50, 7433-7447.

(8) A. Skandalis and S. Pispas, Polymer, 2018, 157, 9-18.

(9) A. Skandalis and S. Pispas, Polym. Chem, 2017, 8, 4538-4547.

(10) C. Masalles, S. Borrós, C. Viñas and F. Teixidor, Adv. Mater., 2000, 12, 1199-1202.

(11) A. D. Delman, J. J. Kellyt, B. B. Simms and U. S. Naval, J. Polym. Sci. Part A, 1970, 8, 111-123.

(12) M. A. Fox and K. Wade, J. Mater. Chem., 2002, 12, 1301-1306.

(13) T. B. Yisgedu, X. Chen, S. Schricker, J. Parquette, E. A. Meyers and S. G. Shore, Chem. Eur. J., 2009, 15, 2190-2199.

(14) K. R. Wee, Y. J. Cho, S. Jeong, S. Kwon, J. D. Lee, I. H. Suh, S. O. Kang, J. Am. Chem. Soc., 2012, 134, 17982-17990.

(15) A. Ferrer-Ugalde, A. González-Campo, C. Viñas, J. Rodríguez-Romero, R. Santillan, N. Farfán, R. Sillanpää, A. Sousa-Pedrares, R. Núñez and F. Teixidor, Chem. Eur. J., 2014, 20 , 9940-9951.

(16) R. Núñez, I. Romero, F. Teixidor and C. Viñas, Chem. Soc. Rev., 2016, 45, 5147-5173.

(17) C. Bellomo, M. Chaari, J. Cabrera-González, M. Blangetti, C. Lombardi, A. Deagostino, C. Viñas, N. Nerea Gaztelumendi, C. Nogués, R. Nuñez and C. Prandi, Chem. Eur. J., 2018, 24, 15622-15630.

(18) M. Chaari, Z. Kelemen, J. G. Planas, F. Teixidor, D. Choquesillo-Lazarte, A. Ben Salah, C. Viñas and R. Núñez, J. Mater. Chem. C., 2018, 6, 11336-11347.

(19) S. Mukherjee and P. Thilagar, Chem. Commun., 2016, 52, 1070-1093.

(20) J. F. Valliant, K. J. Guenther, A. S. King, P. Morel, P. Schaffer, O. O. Sogbein and K. A. Stephenson, Coord. Chem. Rev., 2002, 232, 173-230.

(21) M. H. Park, K. M. Lee, T. Kim, Y. Do and M. H. Lee, Chem. Asian J., 2011, 6, 1362-1366.

(22) M. Eo, M. H. Park, T. Kim, Y. Do and M. H. Lee, Polymer, 2013, 54, 6321-6328.

(23) G. Vancoillie and R. Hoogenboom, Polymer Chemistry., 2016, 7, 5484-5495.

(24) X. Yin, F. Guo, R. A. Lalancette, F. Jäkle, Macromolecules, 2016, 49, 537-546.

(25) Y. Adachi, Y. Ooyama, Y. Ren, X. Yin, F. Jäkle and J. Ohshita, Polym. Chem., 2018, 9, 291-299.

(26) G. Sun, H. Cui, L.Y. Lin, N.S. Lee, C. Yang, W. L. Neumann, J. N. Freskos, J.J. Shieh, R. B. Dorshow and K. L. Wooley, J. Am. Chem. Soc., 2011, 133, 8534-8543.

(27) O. Grimm, and F. H. Schacher, Polymers, 2018, 10, 645.

(28) A. R. Salgarella, A. Zahoranová, P. Šrámková, M. Majerčíková, E. Pavlova, R. Luxenhofer, J. Kronek, I. Lacík and L. Ricotti, Sci. Rep., 2018, 8, 1-13.

(29) Z. Yinghuai, A. T. Peng, K. Carpenter, J. A. Maguire, N. S. Hosmane and M. Takagaki, J. Am. Chem. Soc., 2005, 127, 9875-9880.

(30) A. González-Campo, A. Ferrer-Ugalde, C. Viñas, F. Teixidor, R. Sillanpää, J. Rodríguez-Romero, R. Santillan, N. Farfán, R. and Núñez, Chem. Eur. J., 2013, 19, 6299-6312.

(31) J. Cabrera-González, A. Ferrer-Ugalde, S. Bhattacharyya, M. Chaari, F. Teixidor, J. Gierschner and R. Núñez, Mater. Chem. C., 2017, 5, 10211-10219.

(32) A. Matuszewska, M. Uchman, A. Adamczyk-Woźniak, A. Sporzyński, S. Pispas, L. Kováčik, M. Štěpánek. Biomacromolecules, 2015, 16, 3731-3739.

(33) M. Štěpánek, J. Hajduová, K. Procházka, M. Šlouf, J. Nebesářová, G. Mountrichas, C. Mantzaridis, S. Pispas. Langmuir, 2012, 28, 307-313.

(34) J. T. Lai, Filla, R. Shea. Macromolecules, 2002, 43, 122-123.

(35) M. R. Hill, R. N. Carmean, S. Sumerlin. Macromolecules, 2015, 48, 5459-5469.

(36) A. Nyström, J. W. Bartels, W. Du, K. L. Wooley. J. Polym. Sci. Part A, 2009, 47, 1023-1037.

(37) T. I. Löbling, O. Ikkala, A. H. Gröschel and A. H. E. Müller, ACS Macro Lett., 2016, 5, 1044-1048.

(38) A. Walther, A. S. Goldmann, R. S. Yelamanchili, M. Drechsler, H. Schmalz, A. Eisenberg and A. H. E. 
Müller, Macromolecules, 2008, 41, 3254-3260.

(39) Y. Mai and A. Eisenberg, Chem. Soc. Rev., 2012, 41, 5969-5985.

(40) A. H. Gröschel, A. H. E. Müller. Nanoscale, 2015, 7, 11841-11876.

(41) R. C. Hayward, D. J. Pochan. Macromolecules, 2010, 43, 3577-3584.

(42) F. T. Meng, G. H. Ma, W. Qiu and Z. G. Su, J. Control. Release, 2003, 91, 407-416.

(43) L. W. Flanagin, C. L. McAdams, W. D. Hinsberg, I. C. Sanchez, and C. G. Willson, Macromolecules, 1999, 32, 5337-5343.

(44) S. Himuro, Polym. J., 1993, 25, 1223-1228. 


\section{Graphical abstract}

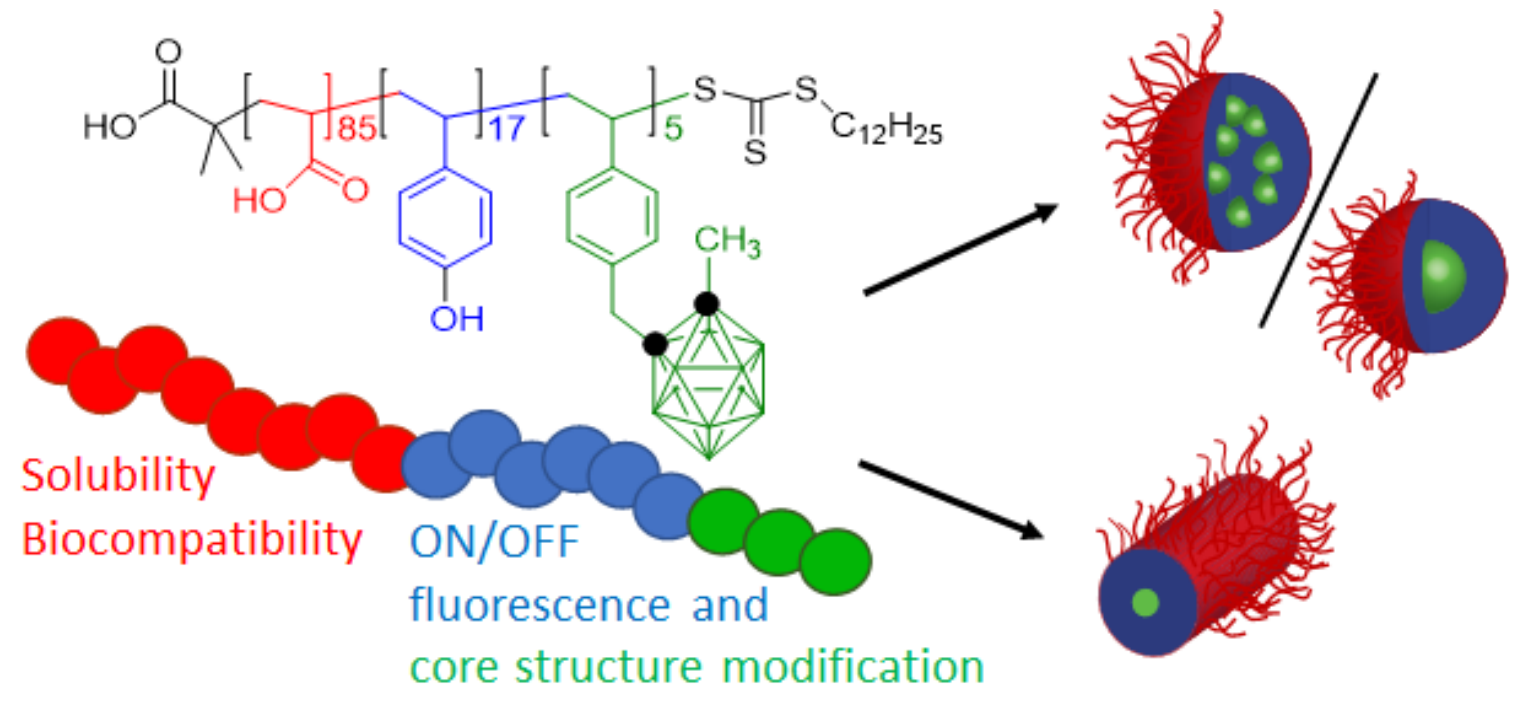

